\title{
Nonlinear description of transversal motion in a laminar boundary layer with streaks
}

\author{
J.A. Martín* and C. Martel ${ }^{\dagger}$ \\ E.T.S.I. Aeronáuticos, Universidad Politécnica de Madrid, Madrid, SPAIN
}

\begin{abstract}
The nonlinear streamwise growth of a spanwise periodic array of steady streaks in a flat plate boundary layer is numerically computed using the well known Reduced NavierStokes formulation. It is found that the flow configuration changes substantially when the amplitude of the streaks grows and the nonlinear effects come into play. The transversal motion (in the wall normal-spanwise plane), which is normally not considered, becomes non-negligible in the nonlinear regime, and it strongly distorts the streamwise velocity profiles, which end up being quite different from those predicted by the linear theory. We analyze in detail the resulting flow patterns for the nonlinearly saturated streaks, and compare them with available experimental results.
\end{abstract}

\section{Introduction}

Streaks naturally develop in a flat plate boundary layer in the presence of small free stream perturbations. They are three dimensional boundary layer flow structures that take the form of spanwise thin and streamwise elongated regions of high speed and low speed flow that alternate in the spanwise direction. The resulting streamwise velocity profile exhibits a strong modulation in the spanwise direction, with a characteristic scale of the order of the width of the boundary layer, and a slow downwards motion in the high speed region and upwards in the low speed region (see Fig. 1).

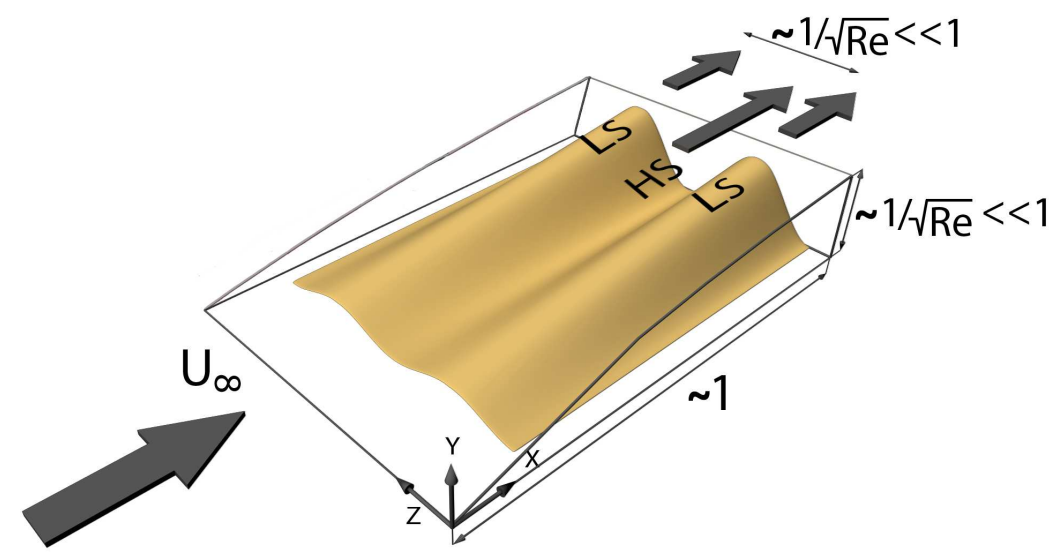

Figure 1. Sketch of the downstream development of a spanwise periodic array of streaks on a flat plate boundary layer, with the asymptotic scaling for $R e \gg 1$ indicated. HS (LS) stands for high (low) streamwise velocity .

Streak computation and analysis is obviously of relevance for the field of fluid mechanics since these are basic flow structures that develop in a canonical problem as it is the flat plate boundary layer. But recently they have become also interesting from the technological point of view. A new strategy has been introduced in order to try to extend the laminar flow region by delaying the transition to turbulence. The idea consists of

*juanangel.martin@upm.es

†carlos.martel@upm.es 
inducing streaks in the laminar boundary layer as a way to improve its stability characteristics and to move further downstream the location of the transition point. This stabilizing effect has been experimentally reproduced using small amplitude streaks, ${ }^{13}$ and stable, intense, fully nonlinear streaks have been also generated experimentally using miniature vortex generators. ${ }^{27}$

Moreover, the edge states for the boundary layer flow, which are receiving so much attention these days, typically include low speed streaky structures. ${ }^{6,7,10}$ These edge states are unstable solutions that live in the boundary of the basin of attraction of the laminar Blasius flow, and somehow mark the threshold for the triggering of the transition to turbulence.

The linear development of small streaks from the Blasius velocity profile was computed in, ${ }^{2,18,29}$ where optimization techniques were applied to the linearized problem in order to locate the maximum streak growth in the streamwise direction. The optimization process is not strictly necessary, as it was reported in, ${ }^{16}$ and the maximum streak growth can be also obtained just by following downstream the only spatially non-decaying mode that comes out from the analysis of the problem in the vicinity of the leading edge. ${ }^{17}$

The linear results indicate that the streaks exhibit transient growth in the streamwise direction (i.e., their amplitude first increases algebraically and then decays to zero to recover the Blasius profile), with the maximum growth taking place for steady streaks and for a particular spanwise wavenumber. ${ }^{2,18,29}$

In the experiments, the algebraic growth of the streaks is, in general, well detected, but there are other results from the linear analysis that show clear quantitative differences with respect to the observations. In the case of streaks excited by free stream turbulence ${ }^{1,21}$ and by energetic turbulent eddies, ${ }^{3,4}$ the measured spanwise scale is approximately half of that predicted by the optimal disturbance computations. And, for streaks forced using an array of small roughness elements placed near the leading edge $\left(\mathrm{see}^{12,30}\right)$, although the general trends are correctly reproduced, there are substantial discrepancies in the downstream development of the streak and in the wall normal disturbance profile for the streamwise velocity, which appears to exhibit two maxima.

In this paper, numerical computations of fully nonlinear streaks are presented that could be helpful in order to understand some of the discrepancies mentioned above. The nonlinear effects, which come into play when the amplitude of the streaks ceases to be negligible, produce a strong distortion the streamwise velocity profile that is not captured in previous linear or small amplitude analyses.

\section{Formulation}

Steady streaks are computed using the so-called Reduced Navier Stokes equations (RNS), which are appropriated for the description of thin streamwise elongated flow configurations and are asymptotically derived from the full 3D Navier Stokes equations (NS) in the limit of large Reynolds number.

The streamwise, wall-normal, and spanwise coordinates, $(X, Y, Z)$, are nondimensionalized using a reference length $L$, and the corresponding velocities $(U, V, W)$ with the stream flow velocity $U_{\infty}$ and the resulting Reynolds number is defined in the usual form, $R e=U_{\infty} L / \nu$, where $\nu$ is the kinematic viscosity. The asymptotic structure of the streaks for $R e \gg 1$ exhibits, as sketched in Fig. 1, slow spatial dependence only in the streamwise direction, and two short spatial scales, in the normal and spanwise direction. The appropriate scaling for the flow variables is given by

$$
\begin{aligned}
& x=X, \quad y=Y \sqrt{R e}, \quad z=Z \sqrt{R e}, \\
& u=U, \quad v=V \sqrt{R e}, \quad w=W \sqrt{R e}, \\
& \text { and } \quad P=p_{0}+p / R e+\ldots,
\end{aligned}
$$

The last equation above indicates that, inside the boundary layer, the pressure deviations from the outer inviscid pressure $P_{\infty}(x)$ has to be small. $P_{\infty}(x)=0$ in the case considered here of a flat plate boundary layer.

Once the expansions in (1) are taken to the 3D steady NS, the full set of RNS is obtained at leading order:

$$
\begin{aligned}
u_{x}+v_{y}+w_{z} & =0 \\
u u_{x}+v u_{y}+w u_{z} & =-P_{\infty}^{\prime}(x)+u_{y y}+u_{z z} \\
u v_{x}+v v_{y}+w v_{z} & =-p_{y}+v_{y y}+v_{z z} \\
u w_{x}+v w_{y}+w w_{z} & =-p_{z}+w_{y y}+w_{z z} .
\end{aligned}
$$


Note that, in contrast with the standard 3D boundary layer equations, ${ }^{25}$ now the higher order $y$ and $z$ momentum equations are required to complete the formulation of the problem, and the correction term of the pressure, $p$, has to be computed to obtain the solution.

The appropriate boundary conditions for a spanwise periodic array of streaks are periodicity in $z$,

$$
(u, v, w, p)\left(x, y, z+L_{z}\right)=(u, v, w, p)(x, y, z)
$$

together with no slip at the bottom wall

$$
(u, v, w)=(0,0,0) \quad \text { at } \quad \mathrm{y}=0,
$$

and, at the upper edge of the boundary layer, the velocities must behave as

$$
(u, v, w) \rightarrow\left(U_{\infty}(x),<v>_{z}, 0\right) \quad \text { as } \quad \mathrm{y} \rightarrow \infty
$$

where $U_{\infty}(x)$ is the free stream velocity, which verifies

$$
U_{\infty} \frac{d U_{\infty}}{d x}=-\frac{d P_{\infty}}{d x},
$$

and in the present case it is simply $U_{\infty}=1$.

The boundary condition (5) states the matching of the velocities with their inviscid counterparts in the simple case considered here of no external free stream disturbances. The wall normal velocity $v$ has to become uniform in $z$ for $y \rightarrow \infty$, that is, it has to approach its spanwise mean value $\left\langle v>_{z}=\frac{1}{L_{z}} \int_{0}^{L_{z}} v d z\right.$. The spanwise mean value $\langle v\rangle_{z}$ for $y \rightarrow \infty$ depends only on $x$, and it is just the displacement velocity of the boundary layer. As it happens in the $2 \mathrm{D}$ boundary layer, the displacement velocity is an output of the RNS and it is to be obtained as part of the solution.

The equations (2) together with the boundary conditions (3), (4), (5) and the extra condition to determine the value of the pressure

$$
<p>_{z}=0 \quad \text { at } \quad \mathrm{y}=0
$$

complete the RNS formulation.

The RNS equations have been known for quite some time now (see, e.g., ${ }^{11,24,28}$ ). Their linearized version has been successfully applied to compute the transient growth of small amplitude streaks from the 2D Blasius boundary layer. ${ }^{16-18,29}$ The full RNS formulation has been used to obtain the nonlinear growth of Gortler vortices over a curved wall, ${ }^{14}$ and to analyze the interaction of a slow streamwise vortex and a fast TS wave ( see $^{15}$ and references there in). The RNS have also been extensively used by Goldstein, Wu and co-workers to describe the excitation of streaks through free stream turbulence, see e.g. ${ }^{22,23,31}$ and the references therein (note that they use the name of Boundary Region Equations for the RNS).

The results shown in this paper are obtained by numerically integrating the RNS using a very fast second order BDF marching scheme in $x$ together with a second order finite difference discretization in the transversal plane $(y, z)$. A complete description of the numerical method used to compute nonlinear streaks and the details of the derivation of the RNS can be found in. ${ }^{19,20}$

\section{Nonlinear streak simulations}

The streaks presented in this paper, similarly to those in ${ }^{8,9}$ and, ${ }^{5}$ are computed starting at $x_{0}=0.4$ with the following initial condition:

$$
\left(u^{0}, v^{0}, w^{0}\right)=\left(U_{b}, V_{b}, 0\right)+A_{s 0}\left(u_{p} \cos \left(\beta_{0} z\right), v_{p} \cos \left(\beta_{0} z\right), w_{p} \sin \left(\beta_{0} z\right)\right)
$$

where $\left(U_{b}, V_{b}, 0\right)$ is the Blasius flow, $\left(u_{p}, v_{p}, w_{p}\right)$ is the optimal perturbation profile obtained in, ${ }^{2} \beta_{0}=0.45$ is the corresponding optimal spanwise wavenumber, and $A_{s 0}$ is the initial streak amplitude.

Two streaks, with initial amplitudes $A_{s 0}=0.1$ and $A_{s 0}=0.3$, have been computed. The resulting downstream evolution of the streak amplitude

$$
A_{s}(x)=\frac{1}{2}\left(\max _{y, z}\left(u-U_{b}\right)-\min _{y, z}\left(u-U_{b}\right)\right),
$$


is plotted in Fig. 2. The small intensity streak $A_{s 0}=0.1$ (which is very similar to streak $\mathrm{C}$ in ${ }^{8,9}$ ) shows the typical transient growth shape predicted by the linear theory: initial increase followed by a decay to the Blasius solution. On the other hand, the streak with mild intensity $A_{s 0}=0.3$ exhibits a much faster growth at the beginning, and the location of the maximum moves significantly upstream, as it is found in the experiments. ${ }^{30}$

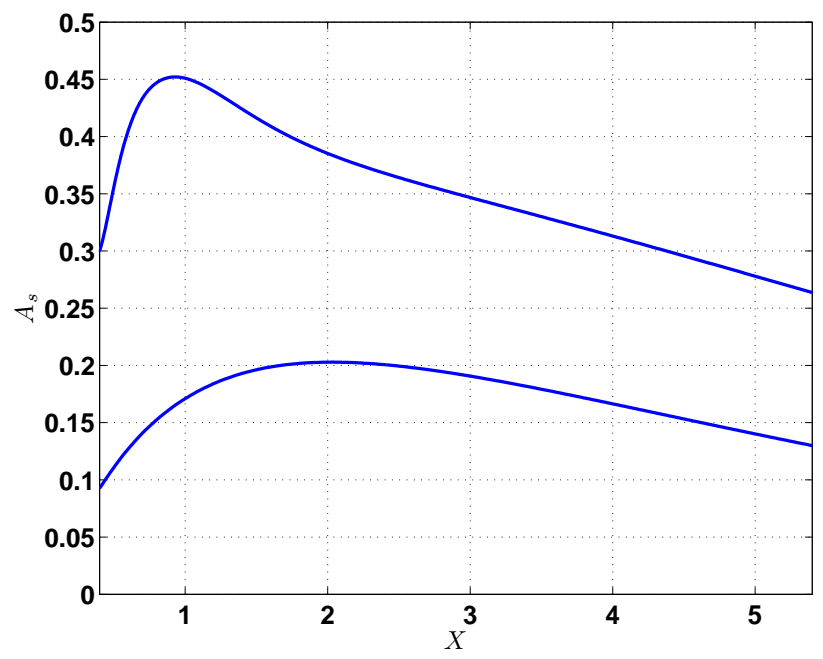

Figure 2. Streamwise evolution of the streak amplitude.

Streaks are basically composed of spanwise alternating regions of faster and slower streamwise flow (see the sketch in Figure 1) but, in order to understand the details of the streak flow structure, the motion in the transversal plane has to be also taken into account. As the flow progresses downstream, the transversal velocities, which are small as compared with the streamwise velocity (recall the scaling in eq. (1)), induce a transversal motion that is not negligible.

The flow structure of the streaks is more clearly appreciated in Fig. 3, which shows the surface of particle trajectories that depart from a horizontal line situated at the beginning of the computational box $(x=0.4$ and $y=3$ ). The small amplitude streak just shows a slight vertical motion that goes up (down) in the slow (fast) streamwise velocity region. But for the streak with $A_{s 0}=0.3$ the situation is quite different, there is an evident effect of the counter rotating motion in the transversal plane that gives these trajectory surfaces a characteristic mushroom-like shape.
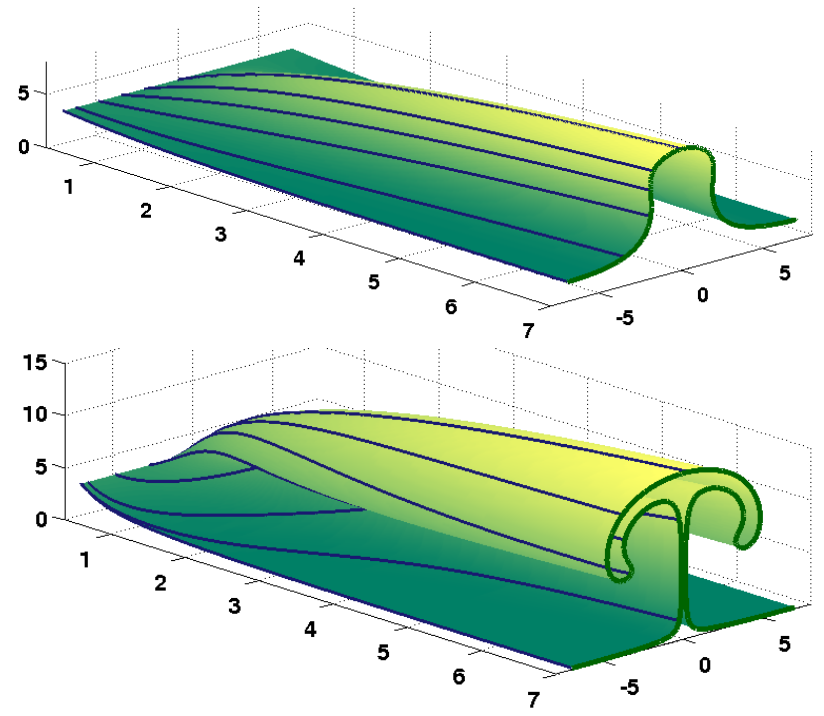

Figure 3. Surfaces of particle trajectories departing from the line $x=0.4, y=3$, for the streaks with initial amplitudes $A_{s 0}=0.1$ (top) and $A_{s 0}=0.3$ (bottom). Several representative particle trajectories are also plotted with blue lines. 

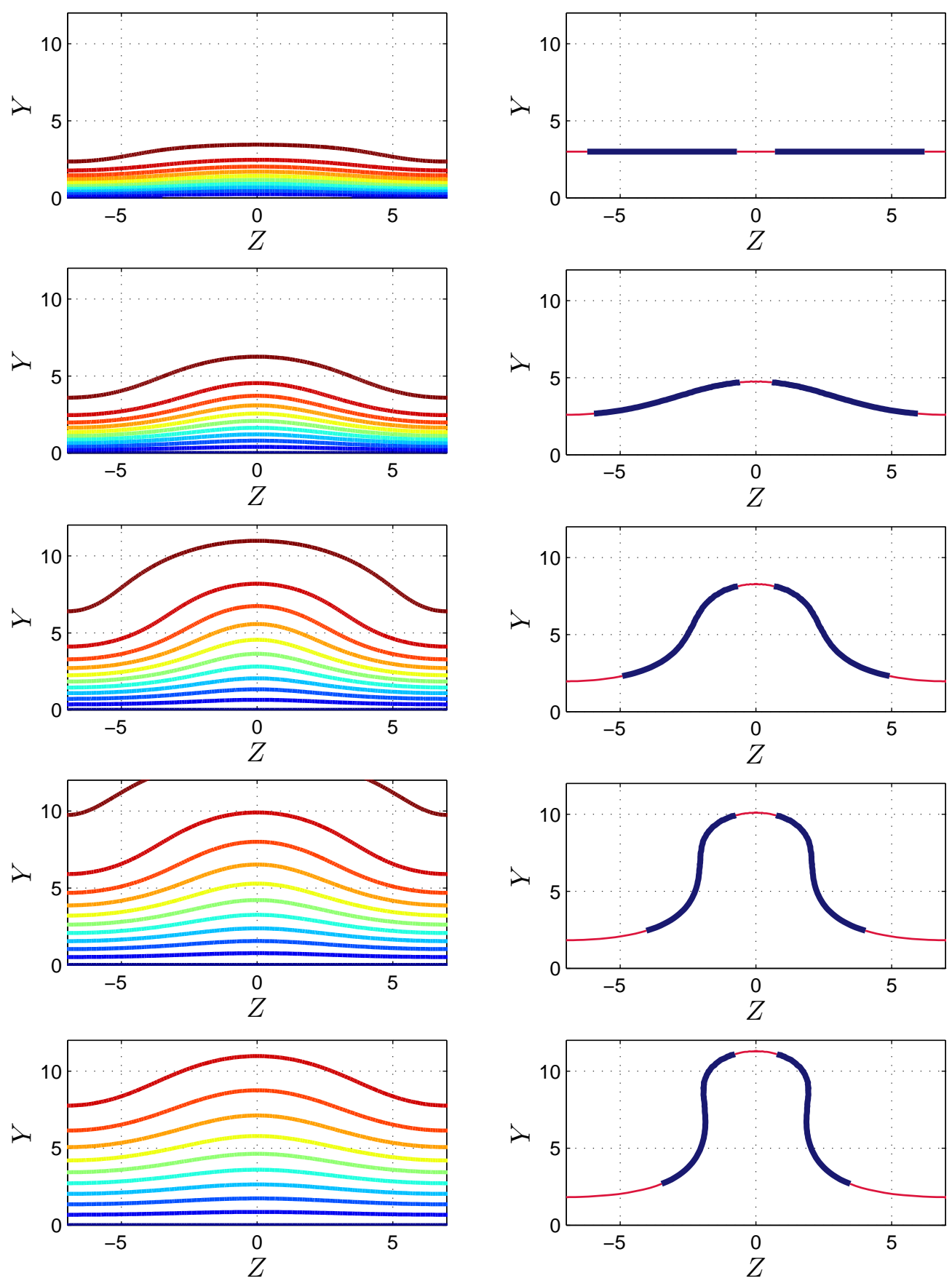

Figure 4. From top to bottom: characteristics of the streak with initial amplitude $A_{s 0}=0.1$ in the transversal plane at $x=0.4,1,3,5$ and 7 . Left: constant streamwise contour lines for $u=0.1,0.2,0.3,0.4,0.5,0.6,0.7,0.8,0.9$ and 0.99 . Right: sections of the particle trajectories starting at the horizontal line $x=0.4, y=3$. 

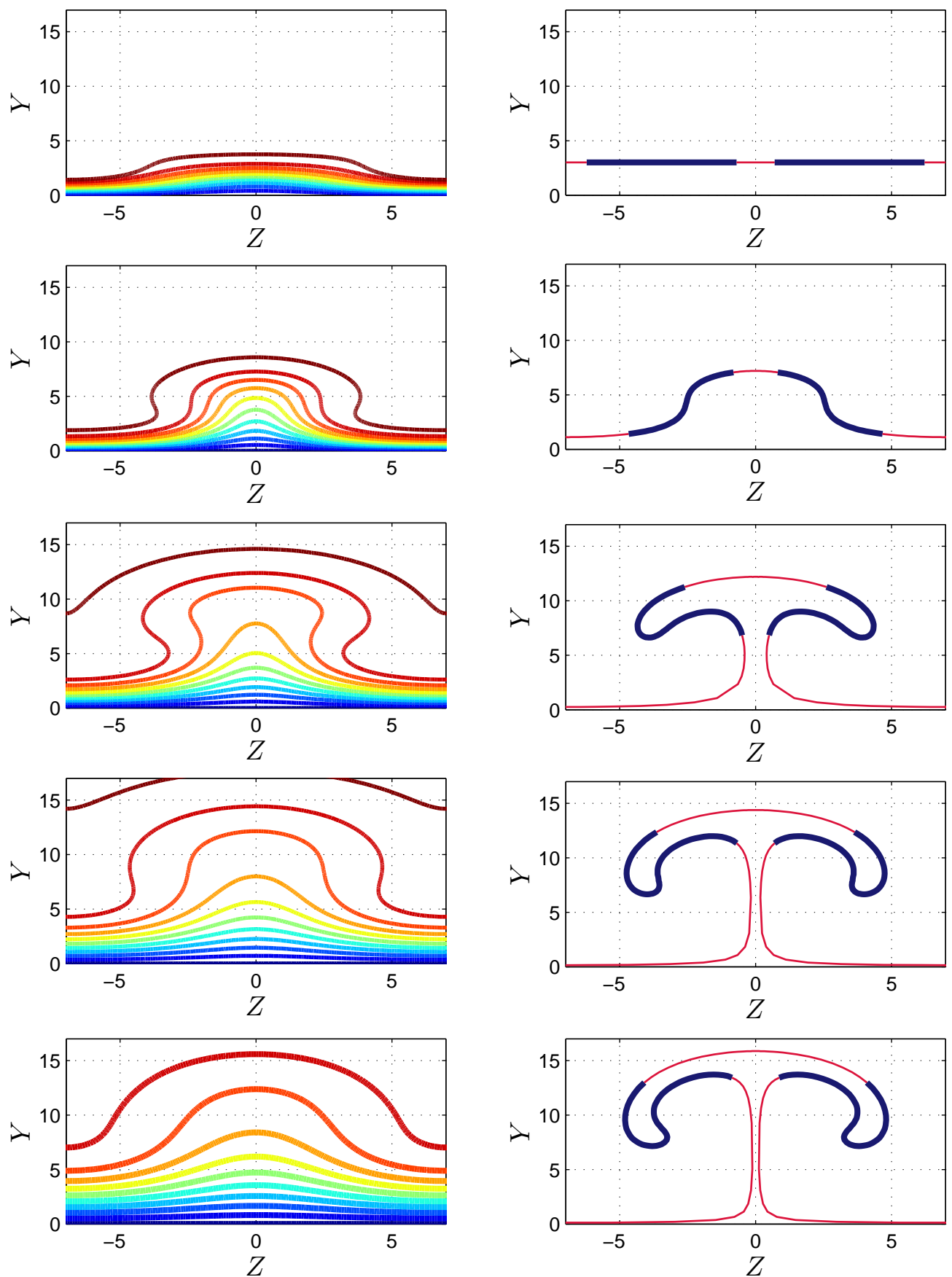

Figure 5. From top to bottom: characteristics of the streak with initial amplitude $A_{s 0}=0.3$ in the transversal plane at $x=0.4,1,3,5$ and 7 . Left: constant streamwise contour lines for $u=0.1,0.2,0.3,0.4,0.5,0.6,0.7,0.8,0.9$ and 0.99 . Right: sections of the particle trajectories starting at the horizontal line $x=0.4, y=3$. 
The transversal structure of the streaks with $A_{s 0}=0.1$ and $A_{s 0}=0.3$ at several streamwise locations is presented in Figs. 4 and 5. The streamwise component of the velocity is plotted in the left column. And, in order to be able to appreciate the accumulation of the particle trajectories, the downstream evolution of the particles trajectories is also shown in the right column, where all particles are marked with a thick line except for the $10 \%$ initially around the center of the box and the $10 \%$ initially near the edges that are plotted using a thin line.

For the $A_{s 0}=0.1$ case (Fig. 4) the evolution is similar to that predicted by the linear theory. The streamwise velocity shows an approximately sinusoidal variation, and the particles just go up in the slow flow region and down in the fast, without any relevant transversal motion.

On the other hand, for the streak with higher amplitude $A_{s 0}=0.3$ (Fig. 5) it can be seen in the right column that, as the flow evolves downstream, the motion in the transversal plane is much more intense, generating a mushroom-like structure. The trajectories strongly drift away from the bottom edges and from the center of the top part: the bottom plot shows that, at $x=7,80 \%$ of the trajectories are curled into two separated roll structures. This rolling of the particle trajectories is an essentially nonlinear effect, which is not captured in the linear approximation, and it becomes more intense as the amplitude of the streak is increased (as it can be seen in the computations presented $\mathrm{in}^{20}$ ).

This concentration of the particles in two counter-rotating rolls would suggest that, in a smoke visualization, one would end up with the smoke accumulated into two separated rolls, giving rise to two smoke traces (instead of one) in the low speed region of a single streak.

In the experiments ${ }^{1,3,4,21}$ top view smoke visualization images of the boundary layer are used to estimate the spanwise period of the streaks. The period is computed as the characteristic spacing between adjacent streamwise smoke traces (i.e., one single smoke trace per streak spanwise period), and the value found is approximately half of that predicted by the optimal disturbance theory. ${ }^{2,18,29}$ As the above results suggest, the possibility of having two smoke traces per spanwise period for a nonlinear streak, could help to understand this quantitative discrepancy between theory and experiments.

The experimental measurements of a streaky boundary layer presented in Fig. 4 of ref. ${ }^{1}$ appear to support this possibility of two smoke traces per streak. They are reproduced in Figs. 6 and 7: Fig. 6 shows a top view of a smoke visualization (flow goes from top to bottom), and the corresponding velocity field at a horizontal section with the average velocity subtracted is plotted with arrows in Fig. 7.

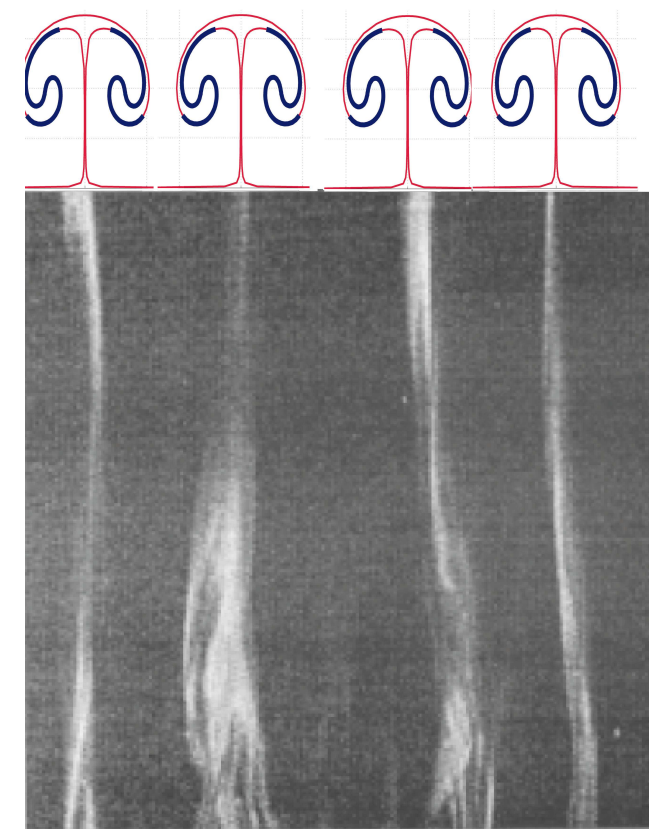

Figure 6. Top view of a streaky boundary layer smoke visualization from ${ }^{1}$ (flow goes from top to bottom), with a sketch above of the corresponding transversal structure for a four streak configuration.

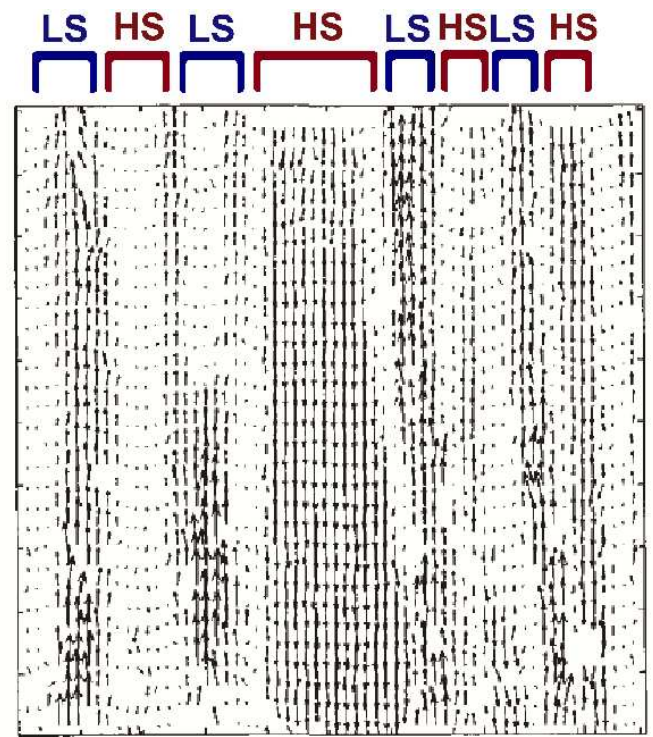

Figure 7. Arrows pointing down (up) indicate mean velocity above (below) the average for the flow shown in Fig. 6 (from $^{1}$ ), with the corresponding regions of high speed (HS) and low speed (LS) marked above for a four streak configuration. 
The interpretation given in ref. ${ }^{1}$ is that there are four streaks in the visualized region. Following this interpretation we have sketched in the top of Fig. 6 the corresponding transversal structure of the four streaks with four mushroom structures, and, since the streaks correspond to spanwise alternating low (LS, arrows pointing up in Fig. 7) and high (HS, arrows pointing down) streamwise velocity structures, we have tried also to approximately mark these alternating LS/HS regions for the four streaks in the top part of Fig. 7.

There is a clear HS region in the center of Fig. 7, but the two adjacent HS regions are much smaller or practically not present at all, as it happens on the left side of the plot where no arrows pointing down can be seen. So, it would not be completely unreasonable to think of a configuration with one HS region in the center of the plot and two wider LS regions on its sides, as it is sketched in the top of Fig. 9. And, in the light of the above results, one could suggest that the appropriate configuration for these visualizations corresponds to two streaks with two smoke traces each in the low speed region, as it is indicated in the top of Figure 8.

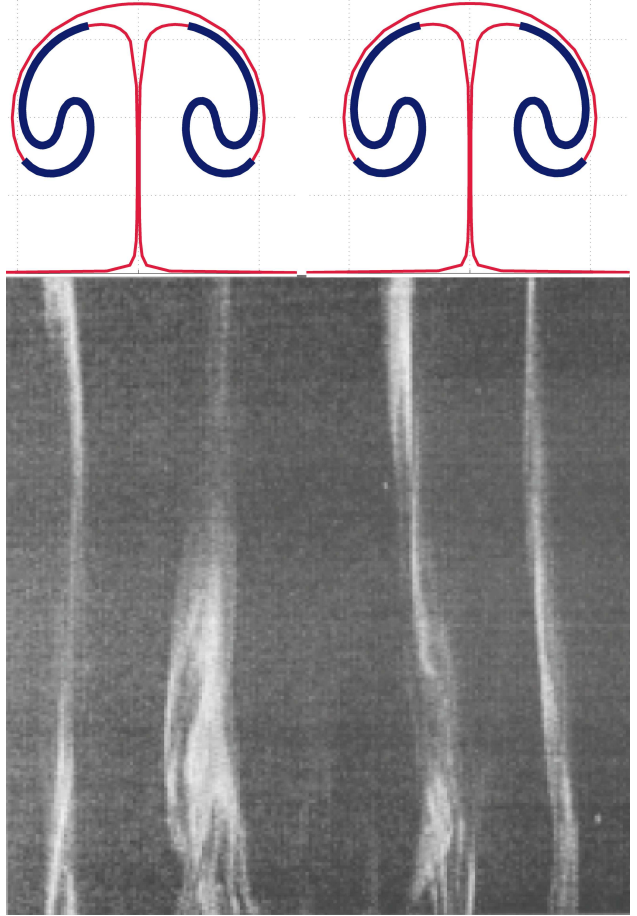

Figure 8. Top view of a streaky boundary layer smoke visualization from ${ }^{1}$ (flow goes from top to bottom), with a sketch above of the corresponding transversal structure for a two streak configuration.

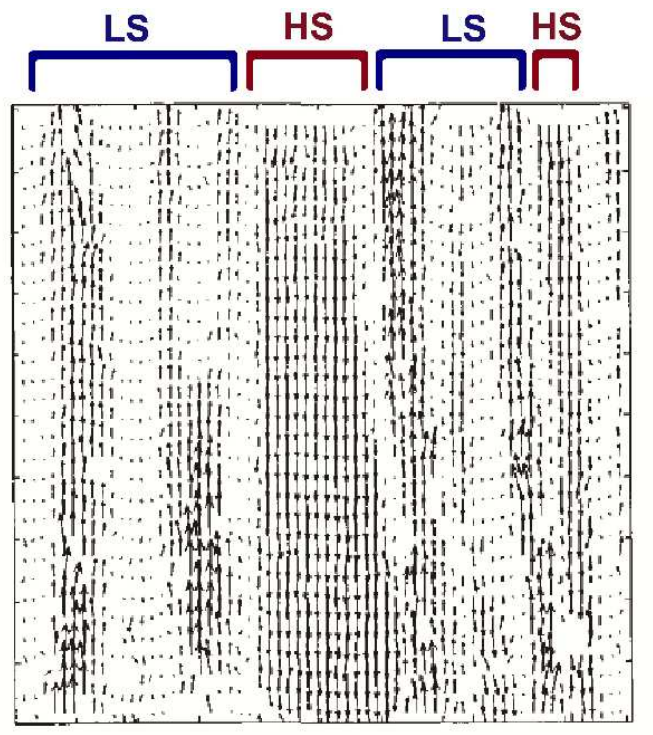

Figure 9. Arrows pointing down (up) indicate mean velocity above (below) the average for the flow shown in Fig. 6 (from $^{1}$ ), with the corresponding regions of high speed (HS) and low speed (LS) marked above for a two streak configuration.

This is of course just a guess that seems quite probable but that definitely would require more experimental work in order to be confirmed. It would be very interesting to know if there is actually the possibility, as the numerics suggest, that the two counter-rotating rolls of the mushroom structure of a high amplitude streak could end up producing two separated smoke traces.

Finally, it is also interesting to point out that the motion in the transversal plane has a strong influence on the streamwise velocity through the nonlinear terms. This is clearly seen in Fig. 10 where the perturbation of the streamwise velocity,

$$
u_{r m s}^{\prime}=\sqrt{\frac{1}{L_{z}} \int_{0}^{L_{z}}\left(u-U_{b}\right)^{2} d z}
$$

is plotted at different downstream locations. The linear theory ${ }^{2,16-18,29}$ predicts $u_{r m s}^{\prime}$ to approximately take the form of the Stewartson mode ${ }^{26}$ with a streamwise varying amplitude. This is basically what is obtained for $A_{s}=0.1$, but for $A_{s}=0.3$ the nonlinear effects are clearly important and distort the profiles, which now exhibit two peaks, and are very similar those reported in the experiment in. ${ }^{30}$ 

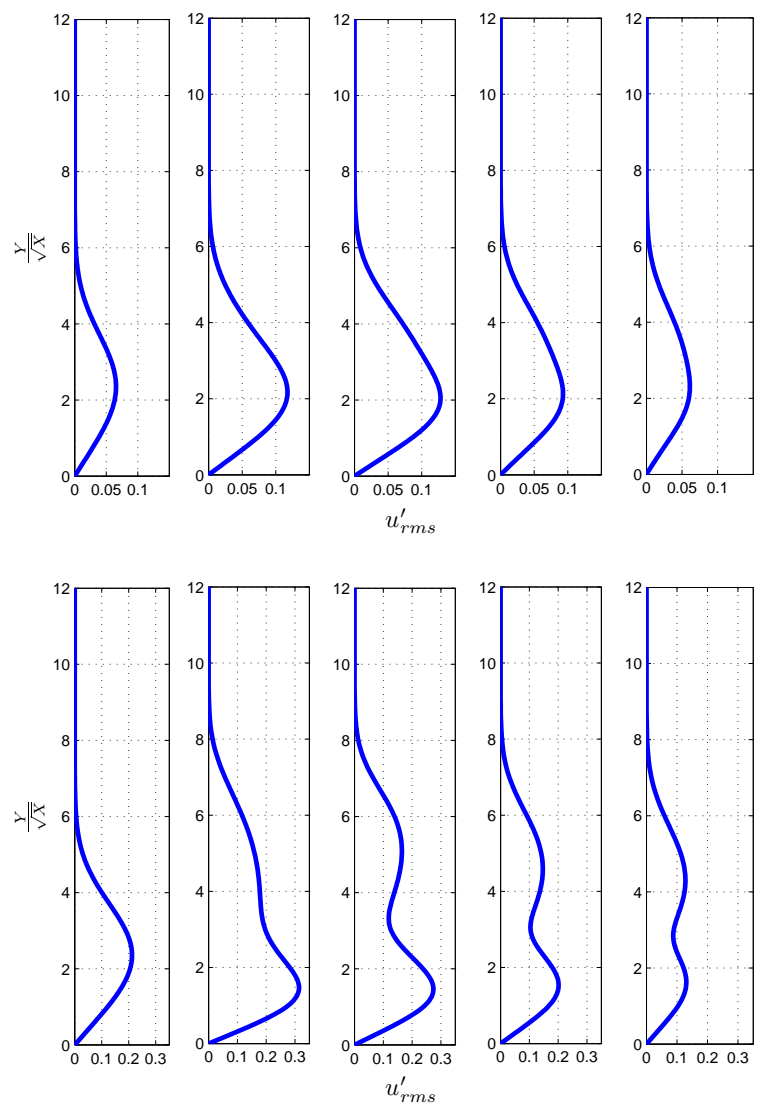

Figure 10. Perturbation of the streamwise velocity at $x=0.4,1,3,5$ and 7 , for the streaks with initial amplitudes $A_{s 0}=0.1$ (top) and $A_{s 0}=0.3$ (bottom).

\section{Concluding remarks}

In the present numerical study, the RNS has been used to describe the downstream evolution of a spanwise periodic array of fully nonlinear streaks in a flat plate boundary layer. The RNS are a set of Reynolds number independent equations that provide the correct asymptotic structure of the streaks in the high Reynolds number limit.

The RNS simulations presented in this paper indicate that the motion in the transversal plane (spanwise and wall normal) is essential to understand the flow configuration of the streak. If the streak amplitude is not small, then, as it progresses downstream, the transversal counter-rotating motion has a strong influence on the streamwise velocity profiles through the convective terms. This is a completely nonlinear effect that is simply not detected in previous linear and small amplitude streak computations. This nonlinear interaction between the transversal motion and the streamwise velocity has been analyzed, and it has been shown that it is clearly present in the available experimental results on laminar streak development in flat plate boundary layers.

An interesting effect detected in the simulations is the possibility, for the high amplitude streaks, to end up generating two separated smoke traces, both in the low speed region of the streak, as a consequence of the motion in the transversal plane. This effect, if it were corroborated experimentally, could help solve the existing discrepancy between the spanwise period of the streaks from the optimal theory and that measured in the experiments.

\section{Acknowledgments}

The authors would like to thank C. Cossu and S. Bagheri for providing access to their results, and to G. Martín for his help with the 3D sketch. This work has been partially supported by the Spanish Ministerio de 


\section{References}

${ }^{1}$ P.H. Alfredsson and M. Matsubara. Free-stream turbulence, streaky structures and transition in boundary layer flow. AIAA paper, 2000-2345, 2000.

${ }^{2}$ P. Andersson, M. Berggren, and D. S. Henningson. Optimal disturbances and bypass transition in boundary layers. Phys. Fluids, 11(1):134-150, 1999.

${ }^{3}$ M. Asai, Y. Konishi, Y. Oizumi, and M Nishioka. Growth and breakdown of low-speed streaks leading to wall turbulence. J. Fluid Mech., 586:371-396, 2007.

${ }^{4} \mathrm{M}$. Asai and M. Nishioka. Boundary-layer transition triggered by hairpin eddies at subcritical reynolds number. J. Fluid Mech., 297:101-122, 1995.

${ }^{5} \mathrm{~S}$. Bagheri and A. Hanifi. The stabilizing effect of streaks on tollmien-schlichting and oblique waves: A parametric study. Phys. Fluids, 19(7), 2007.

${ }^{6}$ D. Biau. Laminar-turbulent separatrix in a boundary layer flow. Phys. Fluids, 24:034107-1-13, 2012.

${ }^{7}$ S. Cherubini, P. De Palma, J. Ch. Robinet, and A. Bottaro. Edge states in a boundary layer. Phys. Fluids, 23:051705-1-4, 2011.

${ }^{8}$ C. Cossu and L. Brandt. Stabilization of tollmien-schlichting waves by finite amplitude optimal streaks in the blasius boundary layer. Phys. Fluids, 14(8):L57-L60, 2002.

${ }^{9}$ C. Cossu and L. Brandt. On tollmien-schlichting-like waves in streaky boundary layers. Eur. J. Mech. B. Fluids, 23(6):815-833, 2004.

${ }^{10}$ Y. Duguet, P. Schlatter, D.S. Henningson, and B. Eckhardt. Self-sustained localized structures in a boundary-layer flow. Phys. Rev. Lett., 108:044501-1-4, 2012.

${ }^{11}$ C. A. J. Fletcher. Computational Techniques for Fluid Dynamics, volume II. Springer Verlag, 1988.

12 J. H. M. Fransson, L. Brandt, A. Talamelli, and C. Cossu. Experimental and theoretical investigation of the nonmodal growth of steady streaks in a flat plate boundary layer. Phys. Fluids, 16(10):3627-3638, 2004.

${ }^{13}$ J. H. M. Fransson, A. Talamelli, L. Brandt, and C. Cossu. Delaying transition to turbulence by a passive mechanism. Phys. Rev. Lett., 96(6):064501-064501, 2006.

${ }^{14} \mathrm{P}$. Hall. The nonlinear development of gortler vortices in growing boundary-layers. J. Fluid Mech., 193:243-266, 1988.

${ }^{15}$ P. Hall and F. T. Smith. On strongly nonlinear vortex wave interactions in boundary-layer-transition. J. Fluid Mech., 227:641-666, 1991.

${ }^{16}$ M. Higuera and J. M. Vega. Modal description of internal optimal streaks. J. Fluid Mech., 626:21-31, 2009.

${ }^{17}$ P. Luchini. Reynolds-number-independent instability of the boundary layer over a flat surface. J. Fluid Mech., 327:101115,1996

${ }^{18}$ P. Luchini. Reynolds-number-independent instability of the boundary layer over a flat surface: optimal perturbations. $J$. Fluid Mech., 404:289-309, 2000.

${ }^{19}$ J.A. Martín and C. Martel. Large reynolds number streak description using rns. In Seventh IUTAM Symposium on Laminar-Turbulent Transition, pages 529-532, 2010.

${ }^{20}$ J.A. Martín and C. Martel. Nonlinear streak computation using boundary region equations. Fluid Dyn. Res., 44:045503, 2012.

${ }^{21}$ M. Matsubara and P. H. Alfredsson. Disturbance growth in boundary layers subjected to free-stream turbulence. $J$. Fluid Mech., 430:149-168, 2001.

${ }^{22} \mathrm{P}$. Ricco. The pre-transitional klebanoff modes and other boundary-layer disturbances induced by small-wavelength free stream vorticity. J. Fluid Mech., 638:267-303, 2009.

${ }^{23} \mathrm{P}$. Ricco, J. Luo, and X. Wu. Evolution and instability of unsteady nonlinear streaks generated by free-stream vortical disturbances. J. Fluid Mech., 677:1-38, 2011.

${ }^{24}$ S. G. Rubin and J. C. Tannehill. Parabolized reduced navier-stokes computational techniques. Annu. Rev. Fluid Mech., $24: 117-144,1992$.

${ }^{25}$ H. Schlichting. Boundary Layer Theory. Mac Graw-Hill, 1968.

${ }^{26} \mathrm{~K}$. Stewartson. On asymptotic expansion in the theory of boundary layers. J. Math. Phys., 39:137-191, 1957.

${ }^{27}$ A. Talamelli and J.H.M. Fransson. High amplitude steady streaks in flat plate boundary layers. AIA A paper, 2010-4291, 2010.

${ }^{28}$ J. C. Tannehill, D. A. Anderson, and R. H. Pletcher. Computational fluid mechanics and heat transfer. Taylor \& Francis, Philadelphia, 2nd edition, 1997.

${ }^{29}$ A. Tumin and E. Reshotko. Spatial theory of optimal disturbances in boundary layers. Phys. Fluids, 13(7):2097-2104, 2001.

${ }^{30}$ E. B. White. Transient growth of stationary disturbances in a flat plate boundary layer. Phys. Fluids, 14(12):4429-4439, 2002.

${ }^{31}$ D.W. Wundrow and M.E. Goldstein. Effect on a laminar boundary layer of small-amplitude streamwise vorticity in the upstream flow. J. Fluid Mech., 426:229-262, 2001. 